\title{
Unique Polyhedral Representations of Continuous Selections for Convex Multiparametric Quadratic Programs
}

\author{
Jørgen Spjøtvold*, Petter Tøndel and Tor A. Johansen \\ Department of Engineering Cybernetics, \\ Norwegian University of Science and Technology, \\ N-7491 Trondheim, Norway. \\ E-mail: \{jorgesp,pettert,torj\}@itk.ntnu.no
}

\begin{abstract}
A method which ensures uniqueness of the polyhedral partition of the parameter space for convex multiparametric quadratic programs $(\mathrm{mp}-\mathrm{QP})$ is presented. When the mp-QP has non-unique solutions, the norm of the solution vector is minimized and a unique affine optimizer function is identified for each region. It is proven, that under certain assumptions on the problem data, a globally continuous piecewise affine optimizer function is selected.

Index Terms-Multiparametric programming, constrained optimal control, quadratic programming, normal cone optimality condition, explicit model predictive control.
\end{abstract}

\section{INTRODUCTION}

Several efficient algorithms for solving multiparametric quadratic programs (mp-QP) have been developed recently [1], [2], [3]. The growing interest in multiparametric problems is due to the fact that explicit solutions to model predictive control problems can be obtained by solving multiparametric programs [1], [4].

Substantial work has been done on the continuity properties of the value function and optimal solution set for multiparametric problems [5], [6], [7], [8], [9], [10]. Continuity of the optimal set mapping is closely related to the stability of the optimization problem and the stability of quadratic programs is studied in [11] and [12]. Continuity and stability results for multiparametric programs are often derived from set theory presented in [13], [14] and [15].

The algorithm presented by Bemporad et al. [1] obtains solutions to strictly convex mp-QPs, however, with some modifications, it can also be used for convex problems [2]. Borrelli et al. [16] proposed a geometric algorithm for multiparametric linear programs (mp-LP) that explores the parameter space in the same manner as in [1]. Common to the algorithms for convex mp-QP, including the geometric algorithm for mp-LP, is that the optimizer function may be discontinuous even if the optimal solution set is a continuous point to set map and therefore admits a continuous selection [17]. Moreover, the polyhedral partition of the parameter space is generally non-unique and may consist of polyhedra with intersecting interiors. A method which ensures continuity of a particular optimizer selection for $\mathrm{mp}$-LPs, without parameters in the objective function, is presented in [18].

\footnotetext{
* Corresponding author.
}

We point out that using the normal cone optimality condition to construct parametric regions of optimality for strictly convex mp-QPs [19] results in a unique polyhedral partition. For convex mp-QP a unique, non-intersecting partition is obtained by always choosing the solution with the least Euclidian norm. If the $\mathrm{mp}-\mathrm{QP}$ has non-unique solutions, a strictly convex mp-QP is formulated such that the norm of the solution vector is minimized subject to the optimality conditions of the original problem.

A unique, non-intersecting polyhedral partition of the parameter space ensures that the polyhedra in the piecewise affine mapping are independent of the algorithm used to explore the parameter space.

If the optimal set mapping for the convex mp-QP is continuous, the minimum norm selection will be a globally continuous mapping from parameter to solution space.

Convex mp-QPs is a problem that arises in explicit softconstrained model predictive control [20], as a sub-problem in mp-NLP algorithms [21], and for explicit model predictive control with a linear cost function [4]. Discontinuities of the optimizer function may lead to chattering in an optimal control approach, and hence, a method which yields a continuous optimizer function is desirable.

\section{PReliminaries}

\section{A. Problem setup}

The standard formulation of an mp-QP is as follows

$$
z^{*}(\theta) \triangleq \min _{x \in \mathbb{R}^{n}}\{f(x, \theta) \mid A x \leq b+S \theta\},
$$

where $f(x, \theta) \triangleq \frac{1}{2} x^{T} H x+\theta^{T} F^{T} x+c^{T} x, \theta \in \mathbb{R}^{s}$ is a parameter of the optimization problem, and the vector $x \in$ $\mathbb{R}^{n}$ is to be optimized for all values of $\theta \in \Theta$, where $\Theta \subseteq$ $\mathbb{R}^{s}$ is some polyhedral set. Moreover $H=H^{T} \in \mathbb{R}^{n \times n}$, $F \in \mathbb{R}^{n \times s}, A \in \mathbb{R}^{q \times n}, b \in \mathbb{R}^{q \times 1}$ and $S \in \mathbb{R}^{q \times s}$ are matrices. If in addition, $H \geq 0$ or $H>0$, the mp-QP is convex or strictly convex, respectively. If $H=0$, then (1) is referred to as a multiparametric linear program (mp-LP), which is a subclass of the problem addressed in this paper.

Recall that the set of affine combinations of points in a set $S \subset \mathbb{R}^{n}$ is called the affine hull of $S$. The dimension of a set $S \subset \mathbb{R}^{n}$, denoted $\operatorname{dim}(S)$; is the dimension of the affine hull of $S$. If the dimension of $S$ is $n$, then $S$ is said to be full-dimensional. 
Let the set of parameters for which the minimum in (1) exists be denoted $\Theta^{*}$ and let $X^{*}(\theta)$ be the set of optimizers to (1) for a given $\theta \in \Theta^{*}$. Without loss of generality it is assumed that $\Theta^{*}$ is full-dimensional, see [16] for details. The feasible set of (1) for a given $\theta \in \Theta$ is given by $\mathcal{P}(\theta) \triangleq$ $\left\{x \in \mathbb{R}^{n} \mid A x \leq b+S \theta\right\}$. We seek the value function $z^{*}$ : $\Theta^{*} \mapsto \mathbb{R}$ and an optimizer function $x^{*}: \Theta^{*} \mapsto \mathbb{R}^{n}$, which is continuous if the problem admits a continuous selection.

We will use the following notation: if $A$ is a matrix, then $A_{i}$ denotes the $i^{\text {th }}$ row of $A$ and $A_{\mathcal{J}}$ denotes the submatrix consisting of the rows of $A$ corresponding to the index set $\mathcal{J}$.

The following definitions are taken from [2] [16].

Definition 1 (Active set): Let $x$ be a feasible solution to (1) for a given $\theta$. We define the active constraints as the set of constraints which fulfill $A_{i} x-b_{i}-S_{i} \theta=0$, and inactive constraints as the set which fulfills $A_{i} x-b_{i}-S_{i} \theta<$ 0 . The active set $\mathcal{A}(x, \theta)$ is the set of indices of the active constraints, that is,

$$
\mathcal{A}(x, \theta) \triangleq\left\{i \in\{1, \ldots, q\} \mid A_{i} x-b_{i}-S_{i} \theta=0\right\} .
$$

Moreover, let $\mathcal{N}(x, \theta)$ denote the set of inactive constraints, that is, $\mathcal{N}(x, \theta) \triangleq\{1, \ldots, q\} \backslash \mathcal{A}(x, \theta)$.

Definition 2 (Optimal active set): Let $\theta$ be given. Let the optimal active set $\mathcal{A}^{*}(\theta)$ be the set of constraints which are active for all $x \in X^{*}(\theta)$, that is

$$
\begin{aligned}
\mathcal{A}^{*}(\theta) & \triangleq\left\{i \mid i \in \mathcal{A}(x, \theta), \forall x \in X^{*}(\theta)\right\} \\
& =\bigcap_{x \in X^{*}(\theta)} \mathcal{A}(x, \theta) .
\end{aligned}
$$

Let $\mathcal{N}^{*}(\theta) \triangleq\{1, \ldots, q\} \backslash \mathcal{A}^{*}(\theta)$.

Definition 3 (LICQ): For an active set $\mathcal{A}$, we say that the linear independence constraint qualification (LICQ) holds if the set of active constraint gradients are linearly independent, i.e., $A_{\mathcal{A}}$ has full row rank.

Definition 4 (Critical region): Given an optimal active set $\mathcal{A}^{*}$ we define the critical region as the set of parameters for which the optimal active set remains unchanged, that is,

$$
\Theta_{\mathcal{A}^{*}} \triangleq\left\{\theta \in \Theta \mid \mathcal{A}^{*}(\theta)=\mathcal{A}^{*}\right\}
$$

It should be noted that critical regions are convex and that their closures are polyhedral. Since the optimal active set is unique for all $\theta \in \Theta^{*}$, critical regions cannot intersect, however, the intersection of their closures may be nonempty. Since $\Theta^{*}$ is assumed to be full-dimensional and the number of optimal active sets is finite, there exists a finite number of full-dimensional critical regions such that the union of their closures is equal to $\Theta^{*}$.

Theorem 1 (Solutions to an $m p-Q P$ ): A solution to a convex mp-QP can be represented as a piecewise affine mapping

$$
x^{*}(\theta)=x_{\mathcal{A}}^{*}(\theta) \text { if } \theta \in R_{\mathcal{A}},
$$

where $R_{\mathcal{A}} \subseteq \Theta^{*}$ are closed, full-dimensional, and convex polyhedral sets such that

$$
\bigcup_{\mathcal{A} \in \mathcal{I}} R_{\mathcal{A}}=\Theta^{*},
$$

and $\mathcal{I}$ is a subset of all possible active sets $\left\{\mathcal{A}\left(x^{*}(\theta), \theta\right) \mid \theta \in \Theta^{*}\right\}$.

Proof: See [1] and [2].

The polyhedra $R_{\mathcal{A}}$ will be defined as described below, and it should be noted that our definitions differ slightly from those in [1] and [2]. Given a $\theta \in \Theta^{*}$ and the associated optimal active set $\overline{\mathcal{A}}^{*}$ such that $\Theta_{\overline{\mathcal{A}}^{*}}$ is full-dimensional. If $X^{*}(\theta)$ is a singleton for all $\theta \in \operatorname{cl}\left(\Theta_{\overline{\mathcal{A}}^{*}}\right)$, then $R_{\overline{\mathcal{A}}^{*}} \triangleq$ $\operatorname{cl}\left(\Theta_{\overline{\mathcal{A}}^{*}}\right)$. If $X^{*}(\theta)$ is not a singleton for all $\theta \in \operatorname{cl}\left(\Theta_{\overline{\mathcal{A}}^{*}}\right)$, then $\Theta_{\overline{\mathcal{A}}^{*}}$ is divided into a finite set of full-dimensional polyhedra, whose union is equal to $\operatorname{cl}\left(\Theta_{\overline{\mathcal{A}}^{*}}\right)$, where each polyhedron is associated with only one affine optimizer function. We will refer to these polyhedra as sub-regions. The optimal solution function $x^{*}(\theta)$ is single valued, since if a given $\theta$ is in more than one $R_{\mathcal{A}}, x_{\mathcal{A}}^{*}(\theta)$ is chosen according to some predetermined ordering of the sets in $\mathcal{I}$.

Definition 5 (Non-intersecting solution): We say that a solution to an mp-QP is non-intersecting if every pair $\left(\mathcal{A}_{i}, \mathcal{A}_{j}\right) \in \mathcal{I} \times \mathcal{I}$ satisfies

$$
\operatorname{dim}\left(R_{\mathcal{A}_{i}} \cap R_{\mathcal{A}_{j}}\right) \leq s-1, i \neq j .
$$

If a solution satisfies Definition 5 , then a given $\theta$ may only be in more than one $R_{\mathcal{A}}$ for lower dimensional subsets of $\Theta^{*}$. Note that closure of a full-dimensional critical region is abbreviated to critical region from this point on.

\section{B. Normal cone optimality condition}

Recall that a set $C$ is called a cone if for every $x \in C$ and scalar $\xi \geq 0$, we have $\xi x \in C$. Moreover, a cone represented by the intersection of a finite number of closed half-spaces is called an $\mathcal{H}$-cone, and a cone represented by a finite number of rays is called a $\mathcal{V}$-cone. If $A$ is a matrix, then cone $(A)$ denotes the set of all nonnegative combinations of the column-vectors of $A$.

Consider the following problem

$$
\begin{aligned}
J^{*} & \triangleq \min _{x \in \mathbb{R}^{n}}\{f(x) \mid x \in \Omega\}, \\
\Omega & \triangleq\left\{x \in \mathbb{R}^{n} \mid g_{i}(x)=0, i \in \mathcal{E} ; g_{j}(x) \leq 0, j \in \mathcal{I}\right\},
\end{aligned}
$$

where $\mathcal{E}$ and $\mathcal{I}$ are finite sets of indices, and $f, g_{i}$ and $g_{j}$ are smooth, real-valued functions on a subset of $\mathbb{R}^{n}$. The following definitions are taken from [22].

Definition 6 (Tangent Vector): A vector $w \in \mathbb{R}^{n}$ is tangent to $\Omega$ at $x$ if for all vector sequences $\left\{x_{i}\right\}$ with $x_{i} \rightarrow x$ and $x_{i} \in \Omega$, and all positive scalar sequences $t_{i} \downarrow 0$, there is a sequence $w_{i} \rightarrow w$ such that $x_{i}+t_{i} w_{i} \in \Omega$ for all $i$.

Definition 7 (Tangent Cone): The tangent cone $T_{\Omega}(x)$ is the collection of all tangent vectors to $\Omega$ at $x$.

Definition 8 (Normal Cone): The normal cone to $\Omega$ at $x$, $N_{\Omega}(x)$, is the orthogonal complement of the tangent cone, that is

$$
N_{\Omega}(x)=\left\{v \mid v^{T} w \leq 0, \quad \forall w \in T_{\Omega}(x)\right\} .
$$

Theorem 2 (First order necessary optimality condition): If $x^{*}$ is a local minimizer of $f$ in $\Omega$, then

$$
-\nabla_{x} f\left(x^{*}\right) \in N_{\Omega}\left(x^{*}\right) .
$$

Proof: See [22]. 
If $f(x)$ and $\Omega$ are convex, then $x^{*}$ is a global minimum and (6) is also sufficient.

Given the polyhedron $\Phi=\{x \mid A x \leq b\}$. Let $x_{0}$ be a point on the boundary of $\Phi$. Let $\mathcal{A}_{0}$ be the set of inequalities which are active at $x_{0}$, hence $A_{i} x_{0}=b_{i}$ for $i \in$ $\mathcal{A}_{0}$, and $A_{i} x_{0}<b_{i}$ for $i \notin \mathcal{A}_{0}$. Note that for a polyhedron the tangent cone $T_{\Phi}\left(x_{0}\right)$ at $x_{0}$, is equal to the set of feasible directions at $x_{0}$ [23], i.e.

$$
T_{\Phi}\left(x_{0}\right)=\left\{d \mid A_{\mathcal{A}_{0}} d \leq 0\right\} .
$$

The normal cone at $x_{0}$ is defined as

$$
N_{\Phi}\left(x_{0}\right)=\operatorname{cone}\left(A_{\mathcal{A}_{0}}^{T}\right),
$$

i.e. a $\mathcal{V}$-cone with the rows of $A_{\mathcal{A}_{0}}$ defining the rays and a single vertex at the origin. Let the matrix $L$ define the corresponding $\mathcal{H}$-cone, that is

$$
N_{\Phi}\left(x_{0}\right)=\{y \mid L y \leq 0\} .
$$

Note that any normal cone can also be associated with an active set, i.e. $N_{\Phi}(\mathcal{A})=\left\{y \mid L_{\mathcal{A}} y \leq 0\right\}$. The optimality condition (6) becomes

$$
L \nabla_{x} f\left(x_{0}\right) \geq 0 .
$$

\section{STRICTLY CONVEX MP-QP}

If $H>0$ in (1), the problem can be reformulated such that only a quadratic term remains in the objective function [1]. Without loss of generality we use the following formulation for strictly convex mp-QP:

$$
z^{*}(\theta) \triangleq \min _{x \in \mathbb{R}^{n}}\left\{\frac{1}{2} x^{T} H x \mid \quad A x \leq b+S \theta\right\} .
$$

In this section it is pointed out that constructing the regions in the solution as proposed in [19] yields a unique set of polyhedra for strictly convex mp-QP. Let $\mathcal{A}^{*}\left(\theta_{0}\right)$ be an optimal active set for (11). The optimizer function associated with $\mathcal{A}^{*}\left(\theta_{0}\right)$ is unique and represented as

$$
x_{\mathcal{A}^{*}}^{*}(\theta)=K \theta+k,
$$

where $K \in \mathbb{R}^{n \times p}$ and $k \in \mathbb{R}^{n}[1]$.

When it is clear from the context, the argument $\theta$ (or $\theta_{0}$ ) will be omitted when referring to an optimal active or inactive set.

Theorem 3 (Critical region): The optimizer function $x_{\mathcal{A}^{*}}^{*}(\theta)$ associated with optimal active set $\mathcal{A}^{*}\left(\theta_{0}\right)$ is optimal in the polyhedron defined by

$$
R_{\mathcal{A}^{*}}=\left\{\begin{array}{l|l}
\theta \in \Theta & \begin{array}{l}
A_{\mathcal{N}^{*}} x_{\mathcal{A}^{*}}^{*}(\theta) \leq S_{\mathcal{N}^{*}} \theta+b_{\mathcal{N}^{*}} \\
L_{\mathcal{A}^{*}} H x_{\mathcal{A}^{*}}^{*}(\theta) \geq 0
\end{array}
\end{array}\right\},
$$

where $L_{\mathcal{A}^{*}}$ is the normal cone defined by the optimal active set $\mathcal{A}^{*}\left(\theta_{0}\right)$.

Proof: See [19].

Lemma 1 (Uniqueness of the solution): The solution to a strictly convex mp-QP obtained by defining sub-regions as in (13), is unique and satisfies Definition 5.

Proof: Uniqueness of the solution follows directly from uniqueness of $\mathcal{A}^{*}(\theta), x_{\mathcal{A}^{*}}^{*}(\theta)$, and the normal cone $N_{\mathcal{P}(\theta)}$ for all $\theta \in \Theta^{*}$. Since $X^{*}(\theta)$ is a singleton for all values of $\theta \in \Theta^{*}$, all $R_{\mathcal{A}} \mathrm{s}$ in the solution are closures of critical regions. Since critical regions does not intersect, the property in Definition 5 is satisfied.

\section{CONVEX MP-QP}

Consider problem (1). In the rest of the paper $H$ is only restricted to be positive semidefinite and symmetric (this includes $H=0$ ), and consequently, the optimizer for a given $\theta$ may be non-unique. We present a method which selects the optimizer function with minimum Euclidian norm. As in [18], the norm of the solution vector will be minimized subject to optimality conditions of the original problem and it is proven that the resulting solution is unique and non-intersecting.

\section{A. Cone condition for convex $Q P$}

The cone condition was defined for a general class of optimization problems in section II-B. We state the optimality condition explicitly for (1);

$$
L_{\mathcal{A}}(H x+F \theta+c) \geq 0,
$$

where $L_{\mathcal{A}}$ is the normal cone matrix corresponding to the active set $\mathcal{A}\left(x^{*}\left(\theta_{0}\right), \theta_{0}\right)$.

Proposition 1: Consider problem (1). Let $\theta=\theta_{0}$ and $\mathcal{A}^{*}\left(\theta_{0}\right)$ be the optimal active set and denote $\mathcal{P}\left(\theta_{0}\right)$ by $\mathcal{P}_{0}$. For any optimal solution $x^{*}\left(\theta_{0}\right)$ we have

$$
-\left(H x^{*}\left(\theta_{0}\right)+F \theta_{0}+c\right) \in N_{\mathcal{P}_{0}}\left(\mathcal{A}^{*}\left(\theta_{0}\right)\right) .
$$

Proof: For $\mathcal{A}\left(x^{*}\left(\theta_{0}\right), \theta_{0}\right)=\mathcal{A}^{*}\left(\theta_{0}\right)$ this is the normal cone condition for optimality. We must show that (15) holds when $\mathcal{A}\left(x^{*}\left(\theta_{0}\right), \theta_{0}\right) \supset \mathcal{A}^{*}\left(\theta_{0}\right)$. Consider an optimal solution $\bar{x}^{*}\left(\theta_{0}\right)$ for which $\mathcal{A}\left(\bar{x}^{*}\left(\theta_{0}\right), \theta_{0}\right)=\mathcal{A}^{*}\left(\theta_{0}\right)$. Let $\tilde{x}^{*}\left(\theta_{0}\right)$ be optimal such that $\mathcal{A}\left(\tilde{x}^{*}\left(\theta_{0}\right), \theta_{0}\right) \supset \mathcal{A}^{*}\left(\theta_{0}\right)$ is the active set. Noting that $H \bar{x}^{*}\left(\theta_{0}\right)=H \tilde{x}^{*}\left(\theta_{0}\right)$ (follows easily from results in [24]) we get:

$$
\begin{aligned}
& -\left(H \tilde{x}^{*}\left(\theta_{0}\right)+F \theta_{0}+c\right) \\
& \quad=-\left(H \bar{x}^{*}\left(\theta_{0}\right)+F \theta_{0}+c\right) \in N_{\mathcal{P}_{0}}\left(\mathcal{A}^{*}\left(\theta_{0}\right)\right) .
\end{aligned}
$$

\section{B. Enforcing uniqueness of the optimizer function}

Lemma 2: Consider problem (1). For every $\mathcal{A}^{*}\left(\theta_{0}\right)$ there is an associated optimal set mapping $X_{\mathcal{A}^{*}}^{*}: \Theta_{\mathcal{A}^{*}} \mapsto 2^{\mathbb{R}^{n}}$ and the mapping can be represented by

$$
\begin{aligned}
X_{\mathcal{A}^{*}}^{*}(\theta) \triangleq \quad & \left\{x_{\mathcal{A}^{*}}(\theta, p) \mid\right. \\
& x_{\mathcal{A}^{*}}(\theta, p) \triangleq K_{\theta} \theta+K_{p} p+k, \\
& L_{\mathcal{A}^{*}}\left(H x_{\mathcal{A}^{*}}(\theta, p)+F \theta+c\right) \geq 0, \\
& \left.A_{\mathcal{N}^{*}} x_{\mathcal{A}^{*}}(\theta, p) \leq b_{\mathcal{N}^{*}}+S_{\mathcal{N}^{*}} \theta\right\}
\end{aligned}
$$

where $L_{\mathcal{A}^{*}}$ defines a normal cone matrix associated with the optimal active set $\mathcal{A}^{*}\left(\theta_{0}\right), K_{p}$ is a basis for the null space of $A_{\mathcal{A}^{*}}, K_{\theta} \in \mathbb{R}^{n \times s}$ and $k \in \mathbb{R}^{n \times 1}$ are constant matrices given by the active set $\mathcal{A}^{*}\left(\theta_{0}\right)$.

Proof: First part of the proof is to show that

$$
x_{\mathcal{A}^{*}}(\theta, p)=K_{\theta} \theta+K_{p} p+k
$$


defines all solutions to

$$
A_{\mathcal{A}^{*}} x=b_{\mathcal{A}^{*}}+S_{\mathcal{A}^{*}} \theta .
$$

Assume now that $A_{\mathcal{A}^{*}}$ does not have full row rank and let $\operatorname{rank}\left(A_{\mathcal{A}^{*}}\right)=m$. Since there exists at least one solution to (19), the system can be reduced to $m$ equations. Applying QR-decomposition $A_{\mathcal{A}^{*}}=Q\left[\begin{array}{c}R \\ 0\end{array}\right]$ as in [1] we get

$$
\begin{aligned}
R x & =b_{1}+S_{1} \theta \\
0 & =b_{2}+S_{2} \theta
\end{aligned}
$$

where $R \in \mathbb{R}^{m \times n}$ and $\left[\begin{array}{l}b_{1} \\ b_{2}\end{array}\right]=Q^{-1} b_{\mathcal{A}^{*}},\left[\begin{array}{l}S_{1} \\ S_{2}\end{array}\right]=Q^{-1} S_{\mathcal{A}^{*}}$. QR-factorization ensures that $\operatorname{rank}(R)=m$, i.e. $R$ has full row rank. We assume that $b_{2}$ and $S_{2}$ are zero or empty (justified in remark 1).

The null-space method [22] can be applied to systems of equations which have full rank, hence, we have that any solution to (20) can be expressed as the sum of a particular solution and a component in the null space of $R$, that is,

$$
x=Y_{R} x_{Y}+Z_{R} x_{Z}
$$

where $Z_{R}$ is a basis for the null space of $R$, and $R Y_{R}$ and $\left[\begin{array}{ll}Y_{R} & Z_{R}\end{array}\right]$ are non-singular. Since $R Z_{R}=0$ we have

$$
x=Y_{R}\left(R Y_{R}\right)^{-1}\left(b_{1}+S_{1} \theta\right)+Z_{R} x_{Z}
$$

Equation (17) ensures that the negative gradient of (1) is in the normal cone defined by $\mathcal{A}^{*}\left(\theta_{0}\right)$ and the solution is thereby optimal. Due to (17), $x_{\mathcal{A}^{*}}(\theta, p)$ is also feasible.

Remark 1: When $S_{2}$ is non-zero, (21) and the inequalities that ensure feasibility of $x$ define a polyhedron in $\mathbb{R}^{w}, w<s$. As explained in section II-A we are only interested in polyhedra in $\mathbb{R}^{s}$. See [1] for details.

The matrices $Z_{R}$ and $Y_{R}$ in the null space method can be chosen by performing a particular $\mathrm{QR}$-factorization, see [22] for details. We can define the explicit expressions for $K_{\theta}, K_{p}$ and $k$

$$
\begin{aligned}
K_{\theta} & =Y_{R}\left(R Y_{R}\right)^{-1} S_{1}, \\
K_{p} & =Z_{R}, \\
k & =Y_{R}\left(R Y_{R}\right)^{-1} b_{1} .
\end{aligned}
$$

Proposition 2: $K_{\theta}$ and $k$ defined by equation (24) and (26), respectively, are unique for a given active set $\mathcal{A}^{*}\left(\theta_{0}\right)$

Proof: All solutions to equation (19) have the same row space component [25].

Lemma 3: Given an optimal active set $\mathcal{A}^{*}\left(\theta_{0}\right)$ for problem (1) and let the affine function $x_{\mathcal{A}^{*}}(\theta, p)$ be given by (18) with $K_{\theta}, K_{p}$ and $k$ defined as in (24)-(26). The following $\mathrm{mp}-\mathrm{QP}$

$v^{*} \triangleq \min _{p}\left\{\frac{1}{2} x_{\mathcal{A}^{*}}(\theta, p)^{T} x_{\mathcal{A}^{*}}(\theta, p) \mid x_{\mathcal{A}^{*}}(\theta, p) \in X_{\mathcal{A}^{*}}^{*}(\theta)\right\}$,

which is defined for all $\theta \in \Theta_{\mathcal{A}^{*}}$ and where $X_{\mathcal{A}^{*}}^{*}(\theta)$ is the set given by (17), has a unique optimizer $p^{*}(\theta)$, which is a piecewise affine function of $\theta$.
Proof: Problem (27) can be written as

$$
\begin{array}{ll} 
& v^{*}=\min _{p} \frac{1}{2} p^{T} H_{p} p+\theta^{T} F_{\theta p}^{T} p+c_{p}^{T} p+C, \text { (28a) } \\
\text { s.t. } & A_{\mathcal{N}^{*}}\left(K_{\theta} \theta+K_{p} p+k\right) \leq b_{\mathcal{N}^{*}}+S_{\mathcal{N}^{*}} \theta, \quad \text { (28b) } \\
& L_{\mathcal{A}^{*}}\left(H\left(K_{\theta} \theta+K_{p} p+k\right)+F \theta+c\right) \geq 0,(28 \mathrm{c})
\end{array}
$$

where $H_{p}=K_{p}^{T} K_{p}, F_{\theta p}^{T}=K_{\theta}^{T} K_{p}, c_{p}^{T}=k^{T} K_{p}$, and $C$ is a constant term. Since $K_{p}$ has linearly independent columns, $K_{p}^{T} K_{p}>0$, and hence, the mp-QP is strictly convex and the results in the previous section apply.

Corollary 1: Any basis $K_{p}$ for the null space of $A_{\mathcal{A}^{*}}$ in equation (18) will yield the same optimal $x_{\mathcal{A}^{*}}\left(\theta, p^{*}(\theta)\right)$ in problem (27). Moreover, the product $K_{p} p^{*}(\theta)$ is unique regardless of the chosen basis.

Proof: All valid bases $K_{p}$ will define the same convex set $X_{\mathcal{A}^{*}}^{*}(\theta)$. Minimizing the strictly convex function $\frac{1}{2} x_{\mathcal{A}^{*}}^{T}(\theta, p) x_{\mathcal{A}^{*}}(\theta, p)$ must then yield a unique optimal solution $x_{\mathcal{A}^{*}}\left(\theta, p^{*}(\theta)\right)$. Since $K_{\theta}, k$ and $x_{\mathcal{A}^{*}}\left(\theta, p^{*}(\theta)\right)$ are unique regardless of the chosen $K_{p}$, so is $K_{p} p^{*}(\theta)$.

Corollary 2: Let the constraints in (27) be written as

$$
\tilde{A} p \leq \tilde{b}+\tilde{S} \theta,
$$

Given the optimal active set $\tilde{\mathcal{A}}^{*}\left(\theta_{0}\right)$ for problem (27) and the associated solution $p_{\tilde{\mathcal{A}}^{*}}^{*}(\theta) . p_{\tilde{\mathcal{A}}^{*}}^{*}(\theta)$ is optimal in the unique region given by

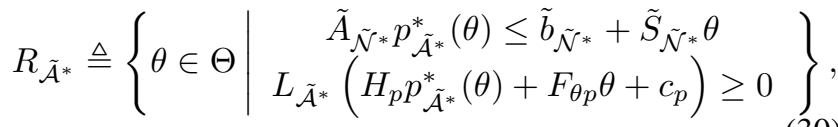

where $L_{\tilde{\mathcal{A}}^{*}}$ is the normal cone matrix defined by the active set $\tilde{\mathcal{A}}^{*}\left(\theta_{0}\right)$.

Proof: The optimizer $x_{\mathcal{A}^{*}}\left(\theta, p^{*}(\theta)\right)$, the optimal active set $\tilde{\mathcal{A}}^{*}(\theta)$, the product $K_{p} p^{*}(\theta)$, and the normal cone $L_{\tilde{\mathcal{A}}^{*}}$ are unique for all $\theta \in \Theta^{*}$, and Lemma 1 applies.

Remark 2: Note that in problem (27) the basis $K_{p}$ is fixed and consequently $p^{*}(\theta)$ is dependent on the choice of the basis. However, since $K_{p} p^{*}(\theta)$ is unique, the region $R_{\tilde{\mathcal{A}}^{*}}$ is uniquely defined for all valid bases.

We define the optimal piecewise affine mapping $x^{*}$ : $\Theta^{*} \mapsto \mathbb{R}^{n}$ as

$$
x^{*}(\theta) \triangleq x_{\mathcal{A}^{*}}\left(\theta, p_{\tilde{\mathcal{A}}^{*}}^{*}(\theta)\right) \quad \text { if } \quad \theta \in R_{\tilde{\mathcal{A}}^{*}} .
$$

Theorem 4 summarizes the approach and concludes that a unique solution that satisfies Definition 5 is obtained.

Theorem 4 (Uniqueness the solution): Let the affine function $x_{\mathcal{A}^{*}}(\theta, p)$ be given by (18) with $K_{\theta}, K_{p}$ and $k$ defined as in (24)-(26) and let $p^{*}(\theta)$ be found from (27). For any $\theta_{0} \in \Theta^{*}$ there is an associated optimal active set $\mathcal{A}^{*}\left(\theta_{0}\right)$ and the affine function $x_{\mathcal{A}^{*}}\left(\theta, p_{\tilde{\mathcal{A}}^{*}}^{*}(\theta)\right)$ is unique and optimal for (1) in the unique polyhedral region given by $R_{\tilde{\mathcal{A}}^{*}}$. Moreover, the piecewise affine mapping $x^{*}: \Theta^{*} \mapsto \mathbb{R}^{n}$ in (31) obtained by defining full dimensional regions as in (30) is an optimal solution to (1), which satisfies Definition 5.

Proof: Uniqueness of $x_{\mathcal{A}^{*}}\left(\theta, p_{\tilde{\mathcal{A}}^{*}}^{*}(\theta)\right)$ is fulfilled by construction and proven in Corollary 1 . Optimality of $x^{*}(\theta)$ with respect to (1) is also fulfilled by construction and 
is a consequence of Lemma 2 and 3. Uniqueness of the region $R_{\tilde{\mathcal{A}}^{*}}$ follows from Corollary 2 . That the solution satisfies Definition 5 follows from uniqueness of $R_{\tilde{\mathcal{A}}^{*}}$.

\section{ON THE CONTINUITY OF SOLUTIONS TO CONVEX MP-QP}

Before we prove that the procedure in section IV-B selects a continuous optimizer function under certain assumptions on the problem data, we restate some definitions from [13]. Let $X$ and $Y$ denote metric spaces in the following three definitions.

Definition 9 (Lower semicontinuous point-to-set map): The point to set map $P: X \mapsto Y$ is lower semicontinuous at $x_{0}$, if for each open set $\Omega$ satisfying $\Omega \cap P\left(x_{0}\right) \neq \emptyset$ there is a neighborhood $U\left(x_{0}\right)$ such that

$$
x \in U\left(x_{0}\right) \Rightarrow P(x) \cap \Omega \neq \emptyset .
$$

Moreover, $P$ is lower semicontinuous on $X$ if it is lower semicontinuous at each $x \in \operatorname{dom}(P)$.

Definition 10 (Upper semicontinuous point-to-set map): The point to set map $P: X \mapsto Y$ is upper semicontinuous at $x_{0}$, if for each open set $\Omega$ containing $P\left(x_{0}\right)$ there exists a neighborhood $U\left(x_{0}\right)$ such that

$$
x \in U\left(x_{0}\right) \Rightarrow P(x) \subset U .
$$

Moreover, $P$ is upper semicontinuous on $X$ if it is upper semicontinuous at each $x \in \operatorname{dom}(P)$.

Definition 11 (Continuous point-to-set map): The point to set map $P: X \mapsto Y$ is continuous at $x_{0}$ if it is both upper semicontinuous and lower semicontinuous at $x_{0}$. It is continuous on $X$ if and only if it is continuous at every $x \in \operatorname{dom}(P)$.

Theorem 5: Consider problem (1) and let the point to set map $X^{*}(\theta)$ be continuous on $\Theta^{*}$. The minimum norm method presented in section IV-B ensures that the optimal mapping $x^{*}: \Theta^{*} \mapsto \mathbb{R}^{n}$ is continuous.

Proof: Let problem (27) be written as

$$
v^{*} \triangleq \min _{x \in \mathbb{R}^{n}}\left\{\frac{1}{2} x^{T} x \mid x \in X^{*}(\theta)\right\}
$$

The minimizer of a strictly convex function over a continuous point to set map is a continuous function [13, Theorem VI.3.3], [26, Corollary 9.3.3].

Since the existence of a continuous selection for problem (1) is ensured by the continuity of $X^{*}(\theta)$ on $\Theta^{*}$ we state the following corollary based on [7, Theorem 3.2.2, Theorem 3.3.3 and Theorem 5.3.2], which shows that $X^{*}(\theta)$ is in fact continuous in some cases.

Corollary 3: The consider problem (1). The point to set map $X^{*}(\theta)$ is continuous on $\Theta^{*}$ if

(i): there does not exist a direction $d \in \mathbb{R}^{n} \backslash\{0\}$ satisfying

$$
H d=0 \text { and }(c+F \theta)^{T} d=0,
$$

$\forall \theta \in \Theta^{*}$, or

(ii): $F=0$.

Proof: Note first that $X^{*}(\theta)$ is upper semicontinuous on $\Theta^{*}$ [7, Theorem 5.3.2]. (i): By [7, Theorem 3.2.3] $X^{*}(\theta)$ is lower semicontinuous on $\Theta^{*}$ if the lineality space

$$
M=\left\{d \in \mathbb{R}^{n} \mid H d=0,(c+F \theta)^{T} d=0, A_{\mathcal{A}^{*}} d=0\right\}
$$

has the same dimension $\forall \theta \in \Theta^{*}$. If $(i)$ holds, then $\operatorname{dim}(M)=0, \forall \theta \in \Theta \supseteq \Theta^{*}$.

(ii): It follows from [7, Theorem 3.3.3] that $X^{*}(\theta)$ lower semicontinuous on $\Theta^{*}$.

Remark 3: Current research is devoted to obtaining weaker conditions on $f(x, \theta)$ and the constraint set for ensuring the lower semicontinuity of the optimal set mapping. Conditions that involve the assumption that a Slater point exists for the constraint set [6], [7] are not applicable since we are concerned with the set $X^{*}(\theta)=\left\{x \in \mathbb{R}^{n} \mid A x \leq\right.$ $\left.b+S \theta, f(x, \theta) \leq z^{*}(\theta)\right\}$.

\section{EXAMPLE}

An example is included to illustrate uniqueness/nonuniqueness of the solution and point ( $i i$ ) of Corollary 3.

Consider the following convex mp-QP:

$$
\begin{aligned}
& z^{*} \triangleq \min _{\{x \in \mathcal{P}(\theta) \mid \theta \in \Theta\}}\left\{\frac{1}{2} x_{4}^{2}-x_{1}-x_{2}-x_{3}+0.5 x_{5}\right\}, \\
& \mathcal{P}(\theta) \triangleq\left\{x \in \mathbb{R}^{5} \mid \begin{array}{rl}
x_{1}+x_{2}+x_{3} & \leq 10-\theta_{1}-\theta_{2} \\
x_{1}-2 x_{2} & \leq 4-\theta_{1}-2 \theta_{2} \\
-x_{1}-2 x_{3} & \leq 3-\theta_{1}-2 \theta_{2} \\
-x_{3}+x_{5} & \leq 2+\theta_{2} \\
-x_{5} & \leq 2+\theta_{1} \\
x_{4}-x_{5} & \leq-\theta_{1} \\
-3 \leq & x_{1} \\
-3 \leq & x_{2} \leq 3 \\
-3 \leq x_{3} \leq 3
\end{array}\right\},
\end{aligned}
$$

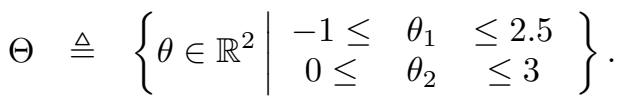

Since $F=0, X^{*}(\theta)$ is continuous on $\Theta^{*}$. The unique set of polytopes obtained by the proposed method is depicted in Figure 1(a), where the subscripts denote which constraints in (1) that are active at the optimum for (27). The constraints are indexed in the order in which they are listed, i.e. $-3 \leq x_{1}$ is constraint number 7 and $x_{1} \leq 3$ is number 8. The optimizer is non-unique in several of the regions in Figure 1(a), and by following the procedure in [2] there are several possible solutions, one of which is depicted in Figure 1(c), where the subscript denotes which constraints that have been arbitrarily selected to construct the regions. Not only is the solution non-unique, but the optimizer is also discontinuous for some selections, see Figure 1(d). By adding $\theta_{1} x_{5}$ to the objective function, neither point $(i)$ nor (ii) in Corollary 3 hold, and consequently $X^{*}(\theta)$ may not be continuous. By following the proposed method, a unique solution is obtained, however, a continuous solution does not exist, see Figures 1(e)-1(f). It should also be noted that when a bilinear term is present in the objective function, algorithms similar to that of [3] are not applicable, since a region may have more than one neighboring region for each facet, see [27] for a discussion of this topic. 

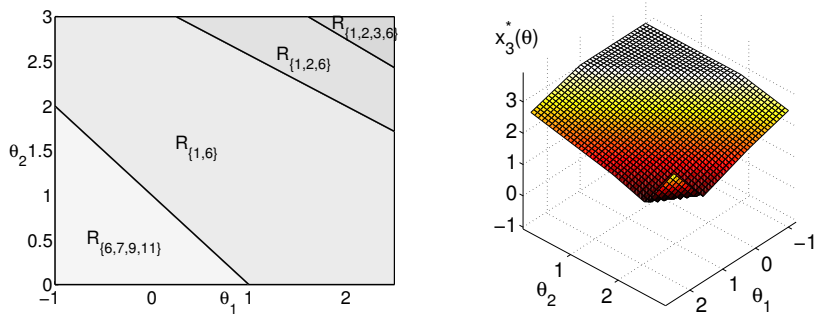

(a) Partition, $F=0$, proposed (b) $x_{3}^{*}(\theta)$ continuous, proposed method. method.
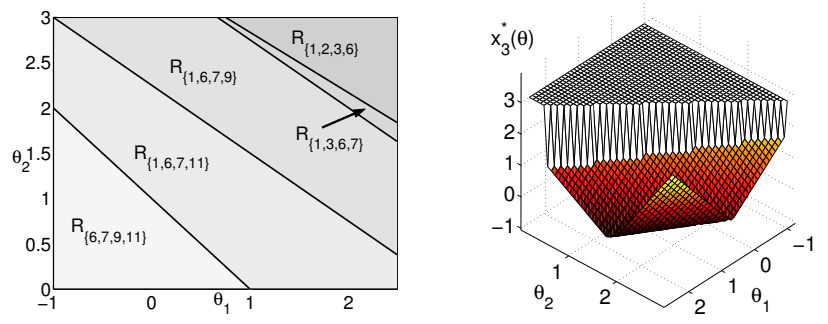

(c) Partition, $F=0$, arbitrary se- (d) $x_{3}^{*}(\theta)$ discontinuous, arbitrary lection.
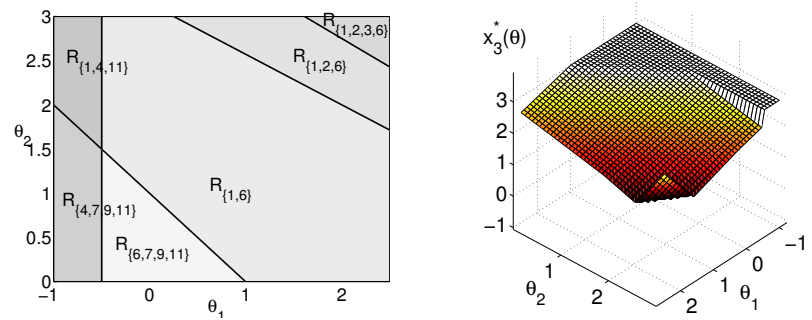

(e) Partition, $F \neq 0$, proposed (f) $x_{3}^{*}(\theta)$ discontinuous, proposed method. method.

\section{CONCLUSIONS}

We have showed that using the normal cone optimality condition to construct critical regions [19] yields a unique and non-intersecting solution for strictly convex mp-QPs. For convex mp-QPs uniqueness of the solution is ensured by choosing the minimum norm optimizer and using the normal cone to characterize the parametric region in which the selection remains optimal.

Global continuity of the piecewise affine optimizer function selected by the proposed method is guaranteed if the optimal set mapping is continuous on the part of the parameter space which renders the solution to the problem feasible and bounded.

\section{ACKNOWLEDGEMENTS}

This research is part of the Strategic University Program on Computational Methods for Nonlinear Motion Control funded by the Research Council of Norway. The second author would like to thank the Research Council of Norway for their support. We also wish to thank Dr. Eric Kerrigan at the Department of Engineering, University of Cambridge, for his comments.

\section{REFERENCES}

[1] A. Bemporad, M. Morari, V. Dua, and E. N. Pistikopoulos, "The explicit linear quadratic regulator for constrained systems," Automatica, vol. 38, no. 1, pp. 3-20, 2002.

[2] P. Tøndel, T. A. Johansen, and A. Bemporad, "Further results on multi-parametric quadratic programming," in Proc. 42nd IEEE Conf. on Decision and Control, Hawaii, 2003, pp. 3173-3178.

[3] M. Baotić, "An efficient algorithm for multi-parametric quadratic programming," ETH Zürich, Institut für Automatik, Physikstrasse 3, CH-8092, Switzerland, Tech. Rep. AUT02-05, 2002.

[4] A. Bemporad, F. Borrelli, and M. Morari, "Model predictive control based on linear programming - the explicit solution," IEEE Trans. on Automatic Control, vol. 47, no. 12, pp. 1974-1985, 2002.

[5] A. V. Fiacco, Introduction to sensitivity and stability analysis in nonlinear programming. Orlando, FL: Academic Press Inc., 1983.

[6] J. Zhao, "The lower continuity of optimal solution sets," Journal of Mathematical Analysis and Applications, vol. 207, pp. 240-250, 1997.

[7] B. Bank, J. Guddat, D. Klatte, B. Kummer, and K. Tammer, Nonlinear Parametric Optimization. Berlin: Birkhäuser, 1983.

[8] M. J. Best and B. Ding, "On the continuity of the minimum in parametric quadratic programs," Journal of Optimization Theory and Applications, vol. 86, no. 1, pp. 245-250, 1972.

[9] W. W. Hogan, "The continuity of the perturbation function of a convex program," Operations Res., vol. 21, no. 1, pp. 351-352, 1973.

[10] _ - "Point-to-set maps in mathematical programming," SIAM Review, vol. 15, no. 3, pp. 591-603, 1973.

[11] M. J. Best and N. Chakravarti, "Stability of linearly constrained convex quadratic programs," Journal of Optimization Theory and Applications, vol. 64, no. 1, pp. 43-53, 1990.

[12] H. X. Phu and N. D. Yen, "On the stability of solutions to quadratic programming problems," Math. Program., vol. 89, pp. 385-394, 2001.

[13] C. Berge, Topological Spaces. London: Oliver and Boyd Ltd, 1963.

[14] G. B. Dantzig, J. Folkman, and N. Z. Shapiro, "On the continuity of the minimum set of a continuous function," Journal of Mathematical Analysis and Applications, vol. 17, no. 3, pp. 519-548, 1967.

[15] F. Hausdorff, Set Theory. New York: Chelsea Publishing Company, 1957.

[16] F. Borrelli, A. Bemporad, and M. Morari, "A geometric algorithm for multi-parametric linear programming," Journal of Optimization Theory and Applications, vol. 118, no. 3, pp. 515-540, 2003.

[17] E. Michaels, "Continuous selections," Annals of Mathematics, vol. 63, pp. 361-382, 1956.

[18] J. Spjøtvold, P. Tøndel, and T. A. Johansen, "A method for obtaining continuous solutions to multiparametric linear programs," in Proc. IFAC World Congress, Prague, 2005, to appear.

[19] D. Q. Mayne and S. Rakovic, "Optimal control of constrained piecewise affine discrete time systems using reverse transformation," in Proc. 41st IEEE Conf. on Decision and Control, vol. 1, Las Vegas, NV, 2002, pp. 1546-1551.

[20] E. C. Kerrigan and J. M. Maciejowski, "Soft constraints and exact penalty functions in model predictive control," in Proc. UKACC International Conference (Control 2000), Cambridge, UK, September 2000.

[21] T. A. Johansen, "On multi-parametric nonlinear programming and explicit nonlinear model predictive control," in Proc. 41st IEEE Conf. on Decision and Control, vol. 3, Las Vegas, NV, 2002, pp. 27682773.

[22] J. Nocedal and S. J. Wright, Numerical Optimization. New York, USA: Springer, 1999.

[23] D. P. Bertsekas, A. Nedic, and A. E. Ozdaglar, Convex Analysis and Optimization. Athena Scientific, 2003.

[24] A. Berkelaar, K. Roos, and T. Terlaky, "The optimal set and optimal partition approach," in Advances in Sensitivity Analysis and Parametric Programming, T. Gal and H. Greenberg, Eds. Kluwer Academic Publishers, 1997, ch. 6, pp. 6.1-6.45.

[25] G. Strang, Linear algebra and its applications, 3rd ed. Florida: Harcourt Brace Jovanovich, 1988.

[26] J. P. Aubin and H. Frankowska, Set-Valued Analysis. Boston: Birkhäuser, 1990.

[27] J. Spjøtvold, E. C. Kerrigan, C. N. Jones, T. A. Johansen, and P. Tøndel, "Conjectures on an algorithm for convex parametric quadratic programs," Deparment of Engineering, University of Cambridge, Cambridge, UK, Tech. Rep. CUED/F-INFENG/TR.496, 2004. 